\title{
Electronic band structure of three-dimensional topological insulators with different stoichiometry composition
}

\author{
I. Grimaldi, ${ }^{1}$ D. Pacilè $\odot,{ }^{1}$ S. V. Eremeev, ${ }^{2,3,4}$ O. De Luca, ${ }^{1,5}$ A. Policicchio $\odot,{ }^{1}$ P. Moras $\odot,{ }^{6}$ P. M. Sheverdyaeva $\odot,{ }^{6}$ \\ A. K. Kundu, ${ }^{6}$ Z. S. Aliev, ${ }^{7}$ P. Rudolf $\odot,{ }^{5}$ R. G. Agostino $\odot,{ }^{1}$ E. V. Chulkov, $, 3,8,9$ and M. Papagno ${ }^{1}$ \\ ${ }^{1}$ Dipartimento di Fisica, Universitá della Calabria, 87036 Arcavacata di Rende (Cs), Italy \\ ${ }^{2}$ Institute of Strength Physics and Materials Science, 634055 Tomsk, Russia \\ ${ }^{3}$ Tomsk State University, Laboratory of Nanostructured Surfaces and Coatings, 634050 Tomsk, Russia \\ ${ }^{4}$ Saint Petersburg State University, Laboratory of Electronic and Spin Structure of Nanosystems, 198504 Saint Petersburg, Russia \\ ${ }^{5}$ Zernike Institute for Advanced Materials, University of Groningen, 9747 AG Groningen, Netherlands \\ ${ }^{6}$ Istituto di Struttura della Materia, Consiglio Nazionale delle Ricerche, 34149 Trieste, Italy \\ ${ }^{7}$ Azerbaijan State Oil and Industry University, Materials Science and Engineering Center, AZ1010 Baku, Azerbaijan \\ ${ }^{8}$ Donostia International Physics Center, 20018 Donostia-San Sebastian, Basque Country, Spain \\ ${ }^{9}$ Departamento de Fisica de Materiales UPV/EHU, Centro de Fisica de Materiales CFM MPC, \\ and Centro Mixto CSICUPV/EHU, 20080 Donostia-San Sebastian, Basque Country, Spain
}

(Received 23 April 2020; revised 15 July 2020; accepted 15 July 2020; published 10 August 2020)

\begin{abstract}
We report on a comparative theoretical and experimental investigation of the electronic band structure of a family of three-dimensional topological insulators, $A^{I V} \mathrm{Bi}_{4} \mathrm{Te}_{7-x} \mathrm{Se}_{x}\left(A^{I V}=\mathrm{Sn}, \mathrm{Pb} ; x=0,1\right)$. We prove by means of density functional theory calculations and angle-resolved photoemission spectroscopy measurements that partial or total substitution of heavy atoms by lighter isoelectronic ones affects the electronic properties of topological insulators. In particular, we show that the modification of the Dirac cone position relative to the Fermi level and the bulk band gap size can be controlled by varying the stoichiometry of the compound. We also demonstrate that the investigated systems are inert to oxygen exposure.
\end{abstract}

DOI: 10.1103/PhysRevB.102.085118

\section{INTRODUCTION}

The theoretical prediction and later experimental verification of the existence of three-dimensional topological insulators (TIs) led to a new compelling field in solid-state physics. TIs are narrow-gap semiconductors with strong spinorbit coupling that is responsible for the appearance of spinpolarized metallic topological states (TSs) with Dirac-type dispersion [1,2]. These peculiar electronic states are topologically protected from backscattering on defects due to time reversal symmetry. These unique electronic properties allow using these materials in spintronics devices [2-6]. Since the discovery of the TI phase in quintuple-layer (QL) structured compounds, the electronic and spin structures of binary-type TIs, such as $\mathrm{Bi}_{2} \mathrm{Se}_{3}, \mathrm{Bi}_{2} \mathrm{Te}_{3}$, and $\mathrm{Sb}_{2} \mathrm{Te}_{3}$, were widely investigated by spin- and angle-resolved photoemission spectroscopy (ARPES) [1,7,8]. Subsequent experiments [9-11] showed that the Dirac state position with respect to the bulk states and the shape of the Dirac cone influences the conduction properties of TIs. Therefore, a method to fine-tune specific properties of the topological insulator such as group velocity, the bulk energy gap, and the position of the Dirac point energy, is desirable. In addition, for practical applications the TS needs to be robust against external agents, whereas it has been shown that adsorption of foreign atoms [12-19] or ultraviolet illumination [20] shifts the Dirac point and modifies the electronic band structure.

One possible way to realize TIs with properties suitable for incorporation in devices is to realize, starting from the well-known binary-based systems, ternary and quaternary layered materials $\left(n A^{I V} B^{V I}-m A_{2}^{V} B_{3}^{V I}\right.$, with $\mathrm{A}^{I V}=\mathrm{Ge}, \mathrm{Sn}, \mathrm{Pb}$; $\left.A^{V}=\mathrm{Bi}, \mathrm{Sb} ; B^{V I}=\mathrm{Te}, \mathrm{Se}\right)$. In contrast to binary compounds, ternary and quaternary TIs are materials made of quintupleor septuple-layer (SL) blocks, as well as sequences of alternating QL and SL units [21-24]. By varying the sequence of the blocks, it is possible, in principle, to design topological insulators with desired electronic properties [25]. Furthermore, first-principles calculations predict that the ternary- and quaternary-based topological compounds allow for modifications of the electronic structure by means of a partial or total substitution of one component with a lighter isoelectronic one [26-28].

Here we compare the electronic properties of one family of three-dimensional topological insulators, $A^{I V} \mathrm{Bi}_{4} \mathrm{Te}_{7-x} \mathrm{Se}_{x}$ $\left(A^{I V}=\mathrm{Sn}, \mathrm{Pb} ; x=0,1\right)$, as determined theoretically by density functional theory (DFT) calculations and experimentally by angle-resolved photoemission spectroscopy. We report evidence of the TSs in the Sn-based topological insulator $\mathrm{SnBi}_{4} \mathrm{Te}_{7}$, which has been theoretically predicted but not experimentally verified $[27,28]$. We demonstrate that partial or total substitution of heavier atoms with lighter isoelectronic ones modifies the electronic structure of these compounds while preserving the exotic properties associated with the topological states.

\section{METHODS}

Single-crystalline ingots of $\mathrm{SnBi}_{4} \mathrm{Te}_{7}$ and $\mathrm{PbBi}_{4} \mathrm{Te}_{6} \mathrm{Se}$ were grown using the modified Bridgman technique. Each polycrystalline compound was synthesized starting from 
(a)

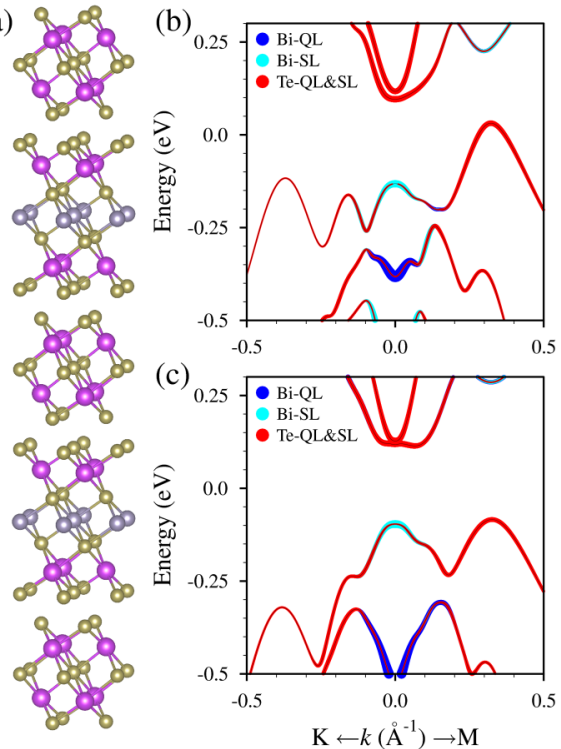

(d) 1.0

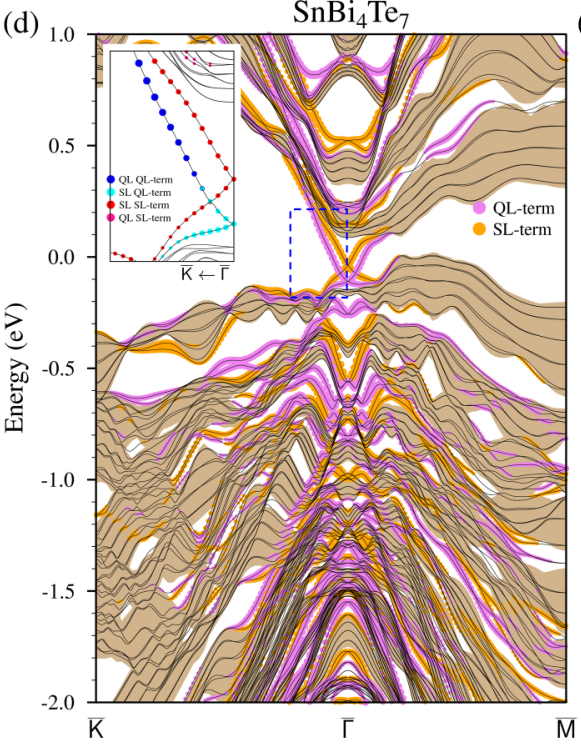

(e)

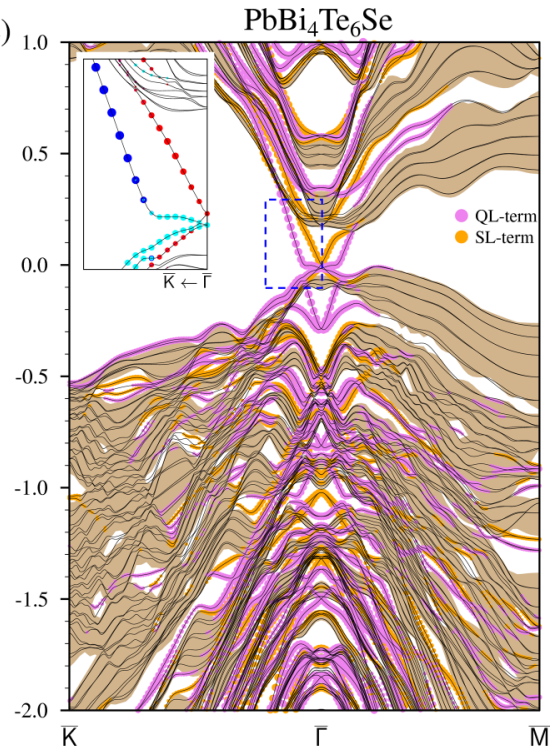

FIG. 1. (a) Atomic structure of $\mathrm{SnBi}_{4} \mathrm{Te}_{7}$. Pink balls represent $\mathrm{Bi}$, yellow balls represent $\mathrm{Te}$, and gray balls represent $\mathrm{Sn}$ atoms. In the crystal structure of $\mathrm{PbBi}_{4} \mathrm{Te}_{6} \mathrm{Se}$ the gray balls correspond to $\mathrm{Pb}$ atoms, and the central atomic layer in the quintuple block is composed of $\mathrm{Se}$ atoms. Bulk electronic structure of (b) $\mathrm{SnBi}_{4} \mathrm{Te}_{7}$ and (c) $\mathrm{PbBi}_{4} \mathrm{Te}_{6} \mathrm{Se}$. The lines are made up of dots whose size denotes the weight of $\mathrm{Bi}$ and Te $p_{z}$ orbitals. Surface electronic structure of (d) $\mathrm{SnBi}_{4} \mathrm{Te}_{7}$ and (e) $\mathrm{PbBi}_{4} \mathrm{Te}_{6} \mathrm{Se}$; the shaded area identifies the bulk-projected bands. The insets in (d) and (e) show a magnified view of the region enclosed by the dotted blue rectangle of the Dirac states, where the size of dots represents the weight of the states in the surface and subsurface building blocks, respectively.

high-purity $(5 \mathrm{~N})$ elements in an evacuated quartz ampoule at about $1000 \mathrm{~K}$ for $8 \mathrm{~h}$ by mixing incessantly. After air cooling, the as-cast sample was placed in a conical-bottom quartz ampoule sealed under a vacuum better than $1 \times 10^{-9}$ mbar. Then, the ampoule was placed in the $\mathrm{T}_{1}$ zone $(\sim 900 \mathrm{~K})$ of a two-zone tube furnace of the Bridgman equipment and held for $8 \mathrm{~h}$ to ensure a complete melting homogenization. The ampoule was then moved from the $\mathrm{T}_{1}$ zone to the $\mathrm{T}_{2}$ zone $(\sim 720 \mathrm{~K})$ at a speed of $1.2 \mathrm{~mm} / \mathrm{h}$. In this way, single crystals with an average size of $\sim 3.5 \mathrm{~cm}$ and a diameter of $\sim 0.8 \mathrm{~cm}$ were obtained. The single-crystalline quality of the as-grown ingots was accurately checked by x-ray diffraction.

All photoemission experiments were carried out at the VUV photoemission beamline of the Elettra synchrotron radiation facility in Trieste. The samples were cleaved in situ in ultrahigh-vacuum conditions (base pressure of $1 \times$ $10^{-10}$ mbar). The high quality of the (0001) fresh cleaved surface was confirmed by the $1 \times 1$ sharp features in the low-energy diffraction pattern. Moreover, the absence of surface contaminants was verified by careful core level photoemission measurements at 700- and 360-eV photon energies. The ARPES measurements were performed at room temperature using a Scienta R-4000 hemispherical electron analyzer, which allows parallel acquisition over a $30^{\circ}$ angular range. Samples were oriented by collecting photoemission intensity maps over an azimuthal angular range of more than $50^{\circ}$ in steps of $1^{\circ}$. The energy and angular resolutions of ARPES were set to $15 \mathrm{meV}$ and $0.3^{\circ}$, respectively. In order to better resolve the topological states features, ARPES was performed at a photon energy $\left(h v=50 \mathrm{eV}\right.$ for $\mathrm{SnBi}_{4} \mathrm{Te}_{7}$ and $h v=30 \mathrm{eV}$ for $\mathrm{PbBi}_{4} \mathrm{Te}_{6} \mathrm{Se}$ ), where the photoemission intensity from the bulk conduction band is strongly reduced. The density functional theory calculations were performed by using the
Vienna Ab initio Simulation Package (VASP) [29,30], with core electrons represented by projector augmented-wave potentials $[31,32]$ with the generalized gradient approximation [33] to the exchange-correlation potential. DFT-D3 van der Waals correction [34] was applied for structure optimization. Spin-orbit interaction was included in structure optimization and electronic band calculations. To simulate surfaces of the $A^{I V} B_{4} \mathrm{Te}_{7-x} \mathrm{Se}_{x}\left(A^{I V}=\mathrm{Sn}, \mathrm{Pb} ; x=0,1\right)$ compounds composed of alternating QL and SL blocks we used asymmetric slabs with QL and SL terminations on the opposite surfaces.

\section{RESULTS AND DISCUSSION}

The primitive cell of the crystal structure of both $\mathrm{SnBi}_{4} \mathrm{Te}_{7}$ and $\mathrm{PbBi}_{4} \mathrm{Te}_{6} \mathrm{Se}$ is hexagonal, with alternating quintuple- and septuple-layer blocks stacked along the $c$ axis, as shown in Fig. 1(a). The bonds inside the SL [Te-Bi-Te-(Pb, Sn)-Te-Sb$\mathrm{Te}$ ] and QL [Te-Bi-(Te, Se)-Bi-Te] have mainly ionic-covalent character, whereas the blocks are held together by weak van der Waals forces $[25,27,28]$.

$\mathrm{SnBi}_{4} \mathrm{Te}_{7}$ and $\mathrm{PbBi}_{4} \mathrm{Te}_{6} \mathrm{Se}$ compounds show a complicated band inversion in the bulk [Figs. 1(b) and 1(c)], where $p_{z}$ orbitals of outer Te atoms of both QL and SL blocks occupy the two lowest conduction bands at $\Gamma$, while $p_{z}$ electrons of $\mathrm{Bi}$ atoms in SL blocks occupy the first and those in QL blocks occupy the second valence bands like in other TIs with alternating QL/SL structure [21].

The bulk band inversion in the studied TIs is different than that in $\mathrm{Bi}_{2} \mathrm{Se}_{3}$-type topological insulators where two bands forming the gap edges participate in the spin-orbitinduced inversion. $\mathrm{SnBi}_{4} \mathrm{Te}_{7}$ and $\mathrm{PbB}_{4} \mathrm{Te}_{6} \mathrm{Se}$ compounds are related to the series of TIs with alternating QL/SL structure, and they have more complicated band inversion in the bulk 
[Figs. 1(b) and 1(c)], involving four bands: two bands from the valence band and two bands from the conduction band. More specifically, the $p_{z}$ orbitals of outer Te atoms of both QL and SL blocks occupy the two lowest conduction bands at $\Gamma$, while $p_{z}$ electrons of $\mathrm{Bi}$ atoms in SL blocks occupy the first and those in QL blocks occupy the second valence bands like in other TIs with alternating QL/SL structure [21].

The calculated band structures with seven- and five-layer block terminations of $\mathrm{SnBi}_{4} \mathrm{Te}_{7}$ and $\mathrm{PbBi}_{4} \mathrm{Te}_{6} \mathrm{Se}$ are presented in Figs. 1(d) and 1(e), respectively. Circles mark the states localized in the outermost blocks, and the shaded area displays the bulk-projected bands. Both compounds are narrow-gap semiconductors. The bulk-projected bands of $\mathrm{SnBi}_{4} \mathrm{Te}_{7}$ show that the conduction-band minimum and valence-band maximum fall along the $\bar{\Gamma}-\bar{M}$ direction and that the bulk band gap is $\sim 100 \mathrm{meV}$ wide, in agreement with an earlier study [27]. Interestingly, the same value is found for the bulk band gap of $\mathrm{PbBi}_{4} \mathrm{Te}_{7}$ [24], implying that the replacement of a light element $(\mathrm{Sn})$ with a heavier isoelectronic one $(\mathrm{Pb})$ in the SL does not necessarily affect the bulk band gap. On the other hand, the bulk band gap in $\mathrm{PbBi}_{4} \mathrm{Te}_{6} \mathrm{Se}$ is 1.5 times larger, proving that the substitution of just one atom (Te) with a lighter one (Se) within the unit cell in the QL has an important impact on the bulk band gap value. This finding is in agreement with recent studies, which have predicted a bulk band gap of $270 \mathrm{meV}$ in $\mathrm{PbBi}_{4} \mathrm{Te}_{3} \mathrm{Se}_{3}$ [35] and of $300 \mathrm{meV}$ in $\mathrm{PbBi}_{4} \mathrm{Te}_{3} \mathrm{~S}_{3}$ [36].

For both compounds the bulk band inversion results in the formation of the single Dirac state on the SL-terminated surface of $\mathrm{SnBi}_{4} \mathrm{Te}_{7}$ [Fig. 1(d)] and $\mathrm{PbBi}_{4} \mathrm{Te}_{6} \mathrm{Se}$ [Fig. 1(e)], completely localized in the surface of the SL block [see insets in Figs. 1(d) and 1(e)], and two surface states on the QL termination. The lowest one arises in the local valence-band gap, which stems wholly from the inversion of the Bi-QL states, whereas the second state resides in the bulk band gap, demonstrating changing localization [Figs. 1(d) and 1(e), insets]. In fact, in the vicinity of the Dirac point this state is localized predominantly in the subsurface SL block, while at larger $k_{\|}$it relocates into the surface QL. The band gap state of the QL-terminated surface of $\mathrm{SnBi}_{4} \mathrm{Te}_{7}$ has a typical Dirac dispersion, characteristic of $\mathrm{Bi}_{2} \mathrm{Te}_{3}$-derived compounds. $\mathrm{PbBi}_{4} \mathrm{Te}_{6} \mathrm{Se}$ instead does not show the deep $\Gamma$ pit in the bulk valence band normally found for $\mathrm{Bi}_{2} \mathrm{Te}_{3}$-based systems, but a pronounced cusp, and the Dirac state has a kinklike dispersion. The latter is similar to the dispersion found in $(\mathrm{SnTe})_{n>1}\left(\mathrm{Bi}_{2} \mathrm{Te}_{3}\right)_{m=1}$ systems [37], where competitive bulk band gap inversion also occurs.

Because of the weak interaction between the layer blocks, the crystals can be easily cleaved, and the surface exposed after cleavage has two possible terminations, the QL or SL block. The cleaved surfaces display sharp and well-defined low-energy electron diffraction patterns with sixfold symmetry (insets of Fig. 2). Since the typical size of both QLand SL-terminated terraces is smaller than the photon beam spot, during an ARPES experiment the band structure of the two terminations can be simultaneously measured. In Fig. 2 we present the second derivative of the photoemission intensity along the energy axis $\left(\frac{\partial^{2} I(E, \boldsymbol{k})}{\partial E^{2}}\right)$ of the wide energy and momentum range dispersions along the $\bar{\Gamma}-\bar{M}$ direction
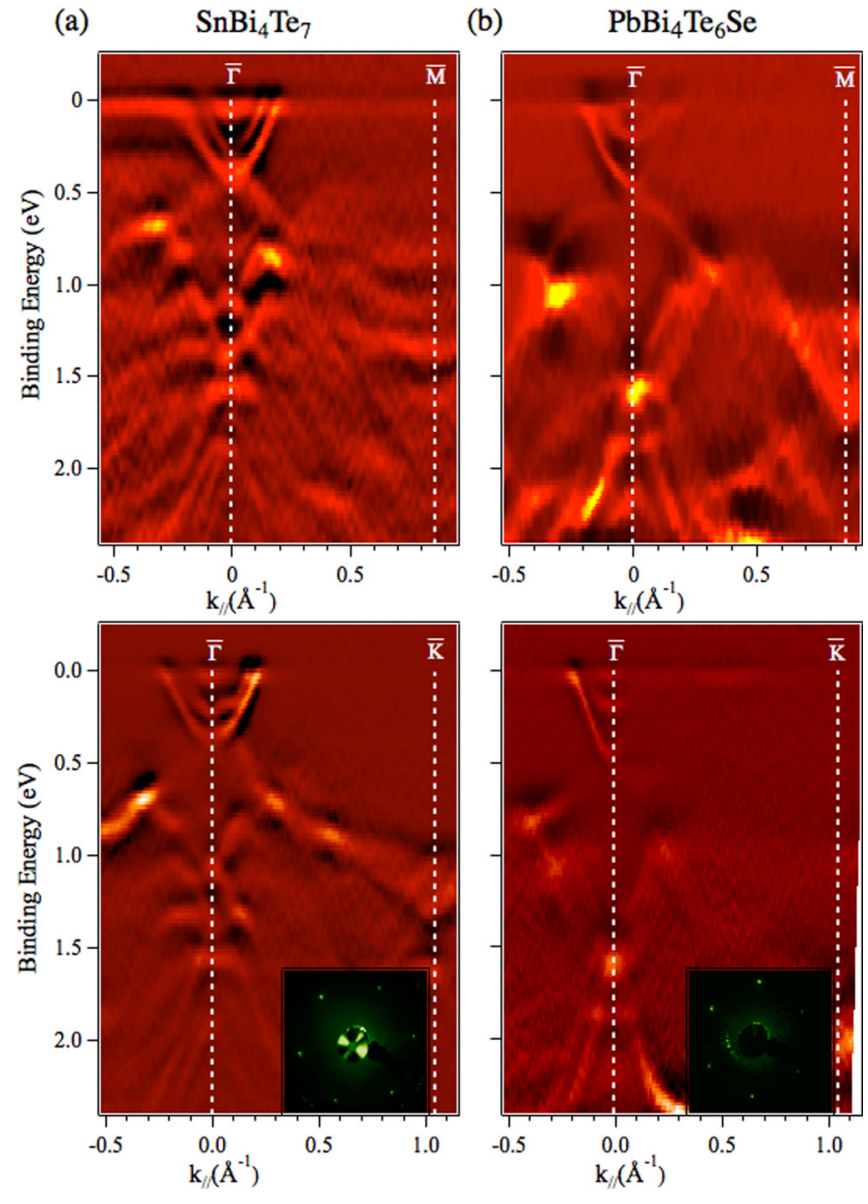

FIG. 2. Second derivative along the energy axis of the energy and momentum dispersions along the $\bar{\Gamma}-\bar{M}$ (top panels) and the $\bar{\Gamma}-\bar{K}$ (bottom panels) directions for (a) $\mathrm{SnBi}_{4} \mathrm{Te}_{7}$ and (b) $\mathrm{PbBi}_{4} \mathrm{Te}_{6} \mathrm{Se}$. The ARPES measurements were recorded at $h v=50 \mathrm{eV}$ photon energy for $\mathrm{SnBi}_{4} \mathrm{Te}_{7}$ and $h v=30 \mathrm{eV}$ for $\mathrm{PbBi}_{4} \mathrm{Te}_{6} \mathrm{Se}$. Insets in the bottom panels are low-electron energy diffraction patterns collected at $55 \mathrm{eV}$ on the same crystals. At this energy the diffraction patterns display nearly sixfold symmetry.

for $\mathrm{SnBi}_{4} \mathrm{Te}_{7}$ [top panel in Fig. 2(a)] and $\mathrm{PbBi}_{4} \mathrm{Te}_{6} \mathrm{Se}$ [top panel in Fig. 2(b)]. In the bottom panels of Figs. 2(a) and 2(b), $\frac{\partial^{2} I(E, \mathbf{k})}{\partial E^{2}}$ along $\bar{\Gamma}-\bar{K}$ for the same two compounds are also shown. These data provide clear evidence of topological states in the energy region between $0.5 \mathrm{eV}$ and $E_{F}$ : linearly dispersing features, i.e., Dirac cone branches intersecting at the $\bar{\Gamma}$ point, can be distinguished. The observed photoelectron intensity asymmetry of the upper Dirac cone branches might be ascribed to the variation of the electron-photon matrix element for the two surface terminations. For both compounds we also observe $M$-shaped bulk bands with an energy maximum of $E_{B} \simeq 0.7 \mathrm{eV}$ and wave number $k_{\|} \simeq \pm 0.14 \AA^{-1}$. Such $\mathrm{M}$-shaped states are due to intrinsic quantization effects [38], also reported for similar compounds, such as $\mathrm{PbBi}_{4} \mathrm{Te}_{7}$ [23], $\mathrm{PbBi}_{6} \mathrm{Te}_{10}$ [25], $\mathrm{PbBi}_{4} \mathrm{Te}_{4} \mathrm{~S}_{3}$ [39], $\mathrm{PbBi}_{4} \mathrm{Te}_{4} \mathrm{Se}_{3}$ [35], and $\mathrm{GeBi}_{4} \mathrm{Te}_{7}[40]$. The diffuse photoemission intensity measured at the $\bar{\Gamma}$ point close to the Fermi level is attributed to partial occupation of the conduction band. 

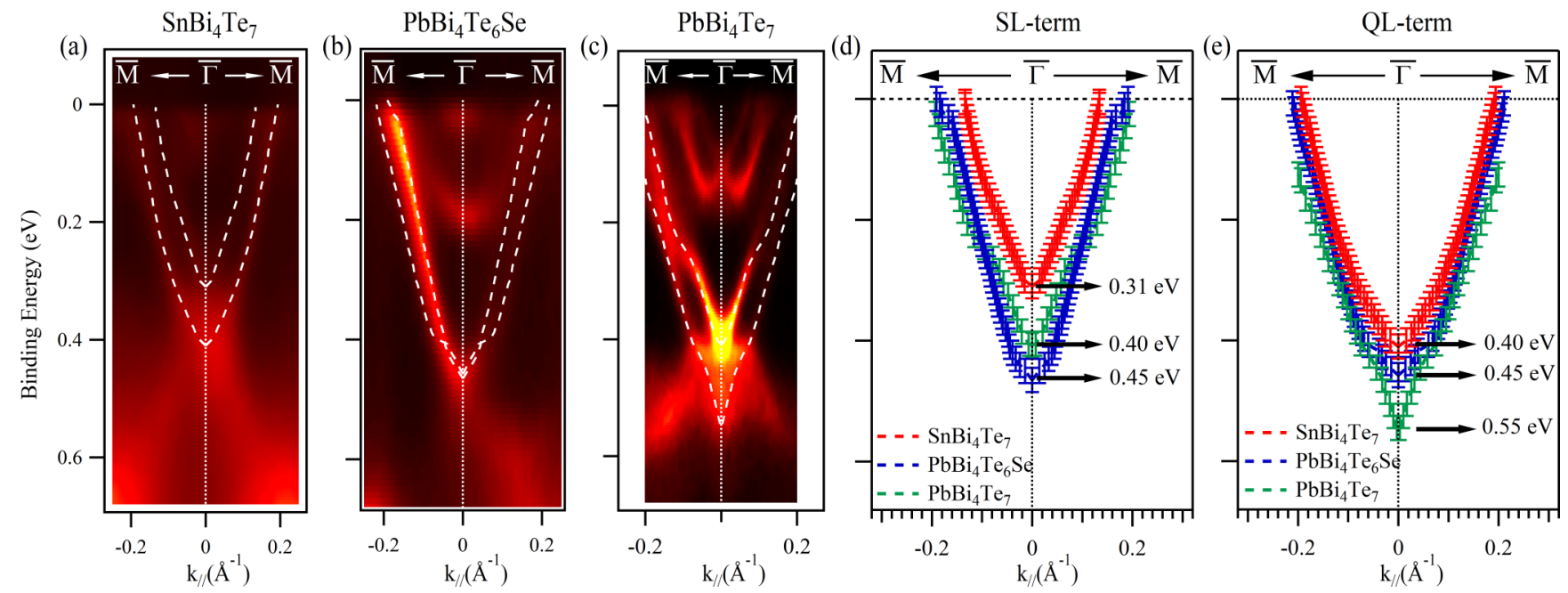

FIG. 3. ARPES measurements along the $\bar{\Gamma}-\bar{M}$ direction of (a) $\mathrm{SnBi}_{4} \mathrm{Te}_{7}$ (recorded at $h v=50 \mathrm{eV}$ ), (b) $\mathrm{PbBi}_{4} \mathrm{Te}_{6} \mathrm{Se}_{(}(h v=30 \mathrm{eV}$ ), and (c) $\mathrm{PbBi}_{4} \mathrm{Te}_{7}(h v=50 \mathrm{eV})$. Dirac state dispersion of the three topological insulators extracted from the maximum peak positions in momentum distribution curves for (d) septuple-layer-terminated and (e) quintuple-layer-terminated surfaces. The same band dispersion has been superimposed on the corresponding spectra [dashed white lines in (a), (b), and (c)].

The experimental bands are in general agreement with the simulated ones in Figs. 1(d) and 1(e). A main difference is the position of the Fermi level, which is predicted to be located close to the Dirac points on both compounds. We note, however, that, in our simulations, the presence of intrinsic impurities or defects, which may alter the relative position of $E_{F}[25,35,39]$, were not taken into account. Furthermore, the calculated valence bands exhibit a maximum along the $\bar{\Gamma}-\bar{M}$ direction, while experimentally, these bands display a diffuse spectral weight intensity for binding energies lower than $\sim 0.8 \mathrm{eV}$ and become fainter as the Dirac energy is approached. The weakness of these features is attributed to the $k_{z}$ dispersion of the electronic states here involved in the photoemission process [39] and to the photon energy used in these ARPES experiments.

To investigate the impact of atom substitution on the Dirac state we collected high-resolution ARPES data, presented in Fig. 3, along the $\bar{\Gamma}-\bar{M}$ direction for $\mathrm{SnBi}_{4} \mathrm{Te}_{7}$ [Fig. 3(a)] and $\mathrm{PbBi}_{4} \mathrm{Te}_{6} \mathrm{Se}$ [Fig. 3(b)] and compared them with those of $\mathrm{PbBi}_{4} \mathrm{Te}_{7}$ [Fig. 3(c)]. The dispersions of the topological states for the SL- and QL-terminated surfaces, determined from the maxima of the momentum distribution curves, are reported in Figs. 3(d) and 3(e), respectively. The derived band dispersions are also superimposed on the corresponding asacquired spectra [Figs. 3(a) and 3(c)].

The ARPES data of all three compounds show two Dirac cones centered at the $\bar{\Gamma}$ point. According to DFT calculations, we assign these features as follows: the outer Dirac cone is related to the QL surface termination, while the inner one corresponds to domains with SL termination. Kinks are observed for $\mathrm{PbBi}_{4} \mathrm{Te}_{6} \mathrm{Se}$. Although the topological states are linear within the gap, in the vicinity of the bulk bands their dispersion usually deviates from the linear behavior due to the crystal field and follows the dispersion of the bulk band edge [22]. These kinks stem from the holelike dispersion of the valence-band edge in the vicinity of the $\bar{\Gamma}$ point. The pronounced cusp in the valence-band dispersion, composed mainly by orbitals of Bi atoms of the SL block, dictates the dispersion of the topological state localizing in the vicinity of the $\bar{\Gamma}$ point within the subsurface SL block. Far from $\bar{\Gamma}$ and the valence-band edge it acquires typical Dirac dispersion, being localized within the surface SL block. Such a change in the spatial localization of the topological state on QL-terminated surfaces is typical for QL/SL-structured TIs and is observed in particular in $\mathrm{PbBi}_{4} \mathrm{Te}_{7}$. However, there the dispersion of the valence-band edge is electronlike, and consequently, the dispersion of the Dirac state deviates from linear dispersion without showing a kink behavior.

For both $\mathrm{SnBi}_{4} \mathrm{Te}_{7}$ and $\mathrm{PbBi}_{4} \mathrm{Te}_{7}$, the overlap of the TS of the QL-terminated terraces with the bulk band dispersions in the energy region $0.40-0.55 \mathrm{eV}$ below the Fermi level does not allow for an accurate determination of the Dirac point energy. Therefore, we estimated the Dirac point energies by linear extrapolation of the data. The group velocity of the Dirac Fermions along the $\bar{\Gamma}-\bar{M}$ direction evaluated sufficiently away from kinks ranges from $4.0 \times 10^{5}$ up to $5.0 \times 10^{5} \mathrm{~m} / \mathrm{s}$ for all compounds. These values are in line with the ones estimated in parental compounds [22,23,39,41,42], suggesting a weak dependence on the stoichiometry of the material.

On the other hand, the data presented in Fig. 3 prove that the Dirac point energy can be significantly shifted by changing the stoichiometry of the crystal. In particular, by replacing the heavier element $(\mathrm{Pb})$ with a lighter isoelectronic atom $(\mathrm{Sn})$ in the SL block, it is possible to shift the topological state of the SL-terminated domain by $0.09 \mathrm{eV}$ toward lower binding energy [Fig. 3(d)]. The topological state of the QL-terminated domain moves instead up by $0.15 \mathrm{eV}$ [Fig. 3(e)]. The substitution of Te atoms with the lighter Se in the central atomic layer of the QL has the most important effect on the QL-terminated TS, shifting it by $100 \mathrm{meV}$ toward lower binding energy [Fig. 3(e)]. The replacement of $\mathrm{Pb}(\mathrm{Te})$ with isoelectronic $\mathrm{Sn}$ (Se) also affects the shape of the Dirac cones and the related position in energy with respect to the continuum of bulk states. 

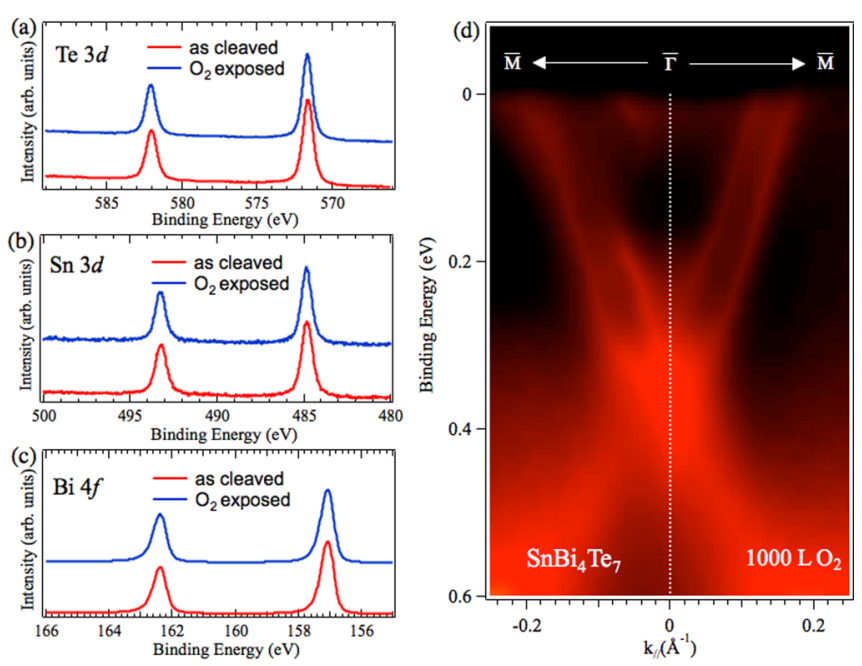

FIG. 4. Photoemission spectra of the (a) Te $3 d$ (recorded at $h v=$ $700 \mathrm{eV}),($ b) $\mathrm{Sn} 3 d(h v=700 \mathrm{eV})$, and (c) Bi $4 f(h v=370 \mathrm{eV})$ core level regions collected on the as-cleaved surface of $\mathrm{SnBi}_{4} \mathrm{Te}_{7}$ before (red curves) and after 1000-L oxygen exposure (blue curves). (d) ARPES spectrum of $\mathrm{SnBi}_{4} \mathrm{Te}_{7}(h v=50 \mathrm{eV})$ after $1000 \mathrm{~L}$ of oxygen.

Finally, there are some contradictory results concerning the stability of topological surface states in ternary and quaternary TIs with respect to external agents (contaminants or impurities). ARPES and core level photoemission investigations of $\mathrm{PbBi}_{4} \mathrm{Te}_{7}$ and $\mathrm{PbBi}_{6} \mathrm{Te}_{10}$ have proved that the Dirac states arising from different surface terminations are unaffected by air exposure [25]. More recently, a spatially selective ARPES study performed on a similar compound, $\mathrm{PbBi}_{4} \mathrm{Te}_{4} \mathrm{~S}_{3}$, reported the vanishing of the QL-related TS after exposure to 100 langmuir $(\mathrm{L})$ of oxygen at room temperature [39]. To shed light on the level of stability of the TSs of this family of compounds, we performed ARPES and core level photoemission measurements on the cleaved surfaces before (red curves in Fig. 4) and after exposure to increasing amounts of oxygen at room temperature, up to $1000 \mathrm{~L}$ on both $\mathrm{SnBi}_{4} \mathrm{Te}_{7}$ (blue curves in Fig. 4) and $\mathrm{PbBi}_{4} \mathrm{Te}_{6} \mathrm{Se}$ (not shown). The photoemission spectra of the Te $3 d$ core level region in Fig. 4(a) show a spin-orbit split doublet with the $3 d_{3 / 2}$ line peaked at $582.05 \mathrm{eV}$ in binding energy and the $3 d_{5 / 2}$ peaked at $571.62 \mathrm{eV}$. No new components or energy shifts were observed after exposure to $1000 \mathrm{~L}$ of oxygen. Likewise, the spectra of the $\mathrm{Sn} 3 d$ core level region presented in Fig. 4(b) (with $3 d_{3 / 2}$ and $3 d_{5 / 2}$ lines peaked at 493.22 and $484.80 \mathrm{eV}$ in binding energy) and of the $\mathrm{Bi} 4 f$ core level region in Fig. 4 (c) (where $4 f_{5 / 2}$ and $4 f_{7 / 2}$ have maxima at 162.38 and $157.06 \mathrm{eV}$ ) do not show any oxygen-induced differences. The TSs do not display identifiable changes upon oxygen exposure, as shown in Fig. 4(d) for $\mathrm{SnBi}_{4} \mathrm{Te}_{7}$. In particular, we did not observe any vanishing of electronic states, energy shifts (within $30 \mathrm{meV}$ ), or new extra electronic states. These measurements prove that the surfaces of the investigated systems are inert to molecular oxygen exposure, preventing modifications or vanishing of the Dirac states.

\section{CONCLUSION}

In this work, we performed a detailed comparative investigation of the electronic structure of ternary and quaternary topological insulators $A^{I V} \mathrm{Bi}_{4} \mathrm{Te}_{7-x} \mathrm{Se}_{x}\left(A^{I V}=\mathrm{Sn}, \mathrm{Pb} ; x=\right.$ $0,1)$ by means of DFT calculations and ARPES measurements. We provided experimental evidence of the topological nature of $\mathrm{SnBi}_{4} \mathrm{Te}_{7}$, displaying two distinct topological states arising from two different surface terminations. Our results on the electronic band structure of $\mathrm{SnBi}_{4} \mathrm{Te}_{7}$ and $\mathrm{PbBi}_{4} \mathrm{Te}_{6} \mathrm{Se}$ compounds compared to that of $\mathrm{PbBi}_{4} \mathrm{Te}_{7}$ demonstrate that partial replacement of heavy atoms (Te) by lighter isoelectronic ones (Se) increases the bulk band gap by a factor of 1.5. We also showed that the shape of the Dirac cones and the related position in energy with respect to the Fermi level can be modified by changing the stoichiometry of the crystal. Finally, we proved that the investigated compounds are inert to $1000 \mathrm{~L}$ of oxygen at room temperature. Our results support an innovative approach that allows for the design of topological insulators with tunable electronic states by varying the stoichiometry of the compounds unaffected by contaminant exposure.

\section{ACKNOWLEDGMENTS}

The authors acknowledge financial support from the Saint Petersburg State University (Grant No. 40990069), the Tomsk State University competitiveness improvement program (Grant No. 8.1.01.2018), the Fundamental Research Program of the State Academies of Sciences (line of research III.23.2.9), and the project EUROFEL-ROADMAP ESFRI. This work was also partly supported by the Italian Ministry of Education, Universities and Research (MIUR) through project PON03PE_00092_1 (EOMAT) and by the Science Development Foundation under the President of the Republic of Azerbaijan (Grant No. EIF/MQM/Elm-Tehsil-1-20161(26)-71/01/4-M-33). S.V.E. acknowledges support from the Russian Science Foundation (Grant No. 18-12-00169) for part of the electronic band structure calculations.
[1] H. Zhang, C.-X. Liu, X.-L. Qi, X. Dai, Z. Fang, and S.-C. Zhang, Nat. Phys. 5, 438 (2009).

[2] M. Z. Hasan and C. L. Kane, Rev. Mod. Phys. 82, 3045 (2010).

[3] L. Fu, C. L. Kane, and E. J. Mele, Phys. Rev. Lett. 98, 106803 (2007).

[4] X.-L. Qi and S.-C. Zhang, Rev. Mod. Phys. 83, 1057 (2011).
[5] R. Roy, Phys. Rev. B 79, 195322 (2009).

[6] D. Hsieh, D. Qian, L. Wray, Y. Xia, Y. S. Hor, R. J. Cava, and M. Z. Hasan, Nature (London) 452, 970 (2008).

[7] D. Hsieh, Y. Xia, D. Qian, L. Wray, F. Meier, J. H. Dil, J. Osterwalder, L. Patthey, A. V. Fedorov, H. Lin, A. Bansil, D. Grauer, Y. S. Hor, R. J. Cava, and M. Z. Hasan, Phys. Rev. Lett. 103, 146401 (2009). 
[8] Y. L. Chen, J. G. Analytis, J.-H. Chu, Z. K. Liu, S.-K. Mo, X. L. Qi, H. J. Zhang, D. H. Lu, X. Dai, Z. Fang, S. C. Zhang, I. R. Fisher, Z. Hussain, and Z.-X. Shen, Science 325, 178 (2009).

[9] W.-C. Lee, C. Wu, D. P. Arovas, and S.-C. Zhang, Phys. Rev. B 80, 245439 (2009).

[10] K. Kuroda, M. Arita, K. Miyamoto, M. Ye, J. Jiang, A. Kimura, E. E. Krasovskii, E. V. Chulkov, H. Iwasawa, T. Okuda, K. Shimada, Y. Ueda, H. Namatame, and M. Taniguchi, Phys. Rev. Lett. 105, 076802 (2010).

[11] R. C. Hatch, M. Bianchi, D. Guan, S. Bao, J. Mi, B. B. Iversen, L. Nilsson, L. Hornekaer, and P. Hofmann, Phys. Rev. B 83, 241303(R) (2011).

[12] H. M. Benia, C. Lin, K. Kern, and C. R. Ast, Phys. Rev. Lett. 107, 177602 (2011).

[13] P. D. C. King, R. C. Hatch, M. Bianchi, R. Ovsyannikov, C. Lupulescu, G. Landolt, B. Slomski, J. H. Dil, D. Guan, J. L. Mi, E. D. L. Rienks, J. Fink, A. Lindblad, S. Svensson, S. Bao, G. Balakrishnan, B. B. Iversen, J. Osterwalder, W. Eberhardt, F. Baumberger, and Ph. Hofmann, Phys. Rev. Lett. 107, 096802 (2011).

[14] T. Valla, Z.-H. Pan, D. Gardner, Y. S. Lee, and S. Chu, Phys Rev. Lett. 108, 117601 (2012).

[15] M. Bianchi, R. C. Hatch, Z. Li, P. Hofmann, F. Song, J. Mi, B. B. Iversen, Z. M. Abd El-Fattah, P. Löptien, L. Zhou, A. A. Khajetoorian, J. Wiebe, R. Wiesendanger, and J. W. Wells, ACS Nano 6, 7009 (2012).

[16] J. Henk, A. Ernst, S. V. Eremeev, E. V. Chulkov, I. V. Maznichenko, and I. Mertig, Phys. Rev. Lett. 108, 206801 (2012).

[17] J. Henk, M. Flieger, I. V. Maznichenko, I. Mertig, A. Ernst, S. V. Eremeev, and E. V. Chulkov, Phys. Rev. Lett. 109, 076801 (2012).

[18] M. Bianchi, D. Guan, S. Bao, J. Mi, B. B. Iversen, P. D. C. King, and P. Hofmann, Nat. Commun. 1, 128 (2010).

[19] P. Ngabonziza, R. Heimbuch, N. de Jong, R. A. Klaassen, M. P. Stehno, M. Snelder, A. Solmaz, S. V. Ramankutty, E. Frantzeskakis, E. van Heumen, G. Koster, M. S. Golden, and H. J. W. Zandvliet, and A. Brinkman, Phys. Rev. B 92, 035405 (2015).

[20] E. Frantzeskakis, S. V. Ramankutty, N. de Jong, Y. K. Huang, Y. P. Pan, A. Tytarenko, M. Radovic, N. C. Plumb, M. Shi, A. Varykhalov, A. de Visser, E. van Heumen, and M. S. Golden, Phys. Rev. X 7, 041041 (2017).

[21] S. V. Eremeev, G. Landolt, T. V. Menshchikova, B. Slomski, Y. M. Koroteev, Z. S. Aliev, M. B. Babanly, J. Henk, A. Ernst, L. Patthey, A. Eich, A. A. Khajetoorians, J. Hagemeister, O. Pietzsch, J. Wiebe, R. Wiesendanger, P. M. Echenique, S. S. Tsirkin, I. R. Amiraslanov, J. H. Dil, and E. V. Chulkov, Nat. Commun. 3, 635 (2012).

[22] K. Kuroda, H. Miyahara, M. Ye, S. V. Eremeev, Yu. M. Koroteev, E. E. Krasovskii, E. V. Chulkov, S. Hiramoto, C. Moriyoshi, Y. Kuroiwa, K. Miyamoto, T. Okuda, M. Arita, K. Shimada, H. Namatame, M. Taniguchi, Y. Ueda, and A. Kimura, Phys. Rev. Lett. 108, 206803 (2012).
[23] M. Papagno, S. V. Eremeev, J. Fujii, Z. S. Aliev, M. B. Babanly, S. Kr. Mahatha, I. Vobornik, N. T. Mamedov, D. Pacilé, and E. V. Chulkov, ACS Nano 10, 3518 (2016).

[24] T. Okuda, T. Maegawa, M. Ye, K. Shirai, T. Warashina, K. Miyamoto, K. Kuroda, M. Arita, Z. S. Aliev, I. R. Amiraslanov, M. B. Babanly, E. V. Chulkov, S. V. Eremeev, A. Kimura, H. Namatame, and M. Taniguchi, Phys. Rev. Lett. 111, 206803 (2013).

[25] D. Pacilè, S. V. Eremeev, M. Caputo, M. Pisarra, O. De Luca, I. Grimaldi, J. Fujii, Z. S. Aliev, M. B. Babanly, I. Vobornik, R. G. Agostino, A. Goldoni, E. V. Chulkov, and M. Papagno, Phys. Status Solidi RRL 12, 1800341 (2018).

[26] T. V. Menshchikova, S. V. Eremeev, Yu. M. Koroteev, V. M. Kuznetsov, and E. Chulkov, JETP Lett. 93, 15 (2011).

[27] M. G. Vergniory, T. V. Menshchikova, S. V. Eremeev, and E. V. Chulkov, Appl. Surf. Sci. 267, 146 (2013).

[28] M. G. Vergniory, T. V. Menshchikova, I. V. Silkin, Yu. M. Koroteev, S. V. Eremeev, and E. V. Chulkov, Phys. Rev. B 92, 045134 (2015).

[29] G. Kresse and J. Hafner, Phys. Rev. B 48, 13115 (1993).

[30] G. Kresse and J. Furthmuller, Phys. Rev. B 54, 11169 (1996).

[31] P. E. Blochl, Phys. Rev. B 50, 17953 (1994).

[32] G. Kresse and D. Joubert, Phys. Rev. B 59, 1758 (1999).

[33] J. P. Perdew, K. Burke, and M. Ernzerhof, Phys. Rev. Lett. 77, 3865 (1996).

[34] S. Grimme, J. Antony, S. Ehrlich, and H. Krieg, J. Chem. Phys. 132, 154104 (2010).

[35] I. A. Shvets, I. I. Klimovskikh, Z. S. Aliev, M. B. Babanly, F. J. Zúñiga, J. Sánchez-Barriga, M. Krivenkov, A. M. Shikin, and E. V. Chulkov, Phys. Rev. B 100, 195127 (2019).

[36] I. V. Silkin, T. V. Menshchikova, M. M. Otrokov, S. V. Eremeev, Yu. M. Koroteev, M. G. Vergniory, V. M. Kuznetsov, and E. V. Chulkov, JETP Lett. 96, 322 (2012).

[37] S. V. Eremeev, T. V. Menshchikova, I. V. Silkin, M. G. Vergniory, P. M. Echenique, and E. V. Chulkov, Phys. Rev. B 91, 245145 (2015).

[38] S. V. Eremeev, M. G. Vergniory, T. V. Menshchikova, A. A. Shaposhnikov, and E. V. Chulkov, New J. Phys. 14, 113030 (2012).

[39] K. Sumida, T. Natsumeda, K. Miyamoto, I. V. Silkin, K. Kuroda, K. Shirai, S. Zhu, K. Taguchi, M. Arita, J. Fujii, A. Varykhalov, O. Rader, V. A. Golyashov, K. A. Kokh, O. E. Tereshchenko, E. V. Chulkov, T. Okuda, and A. Kimura, Phys. Rev. Mater. 2, 104201 (2018).

[40] S. Muff, F. von Rohr, G. Landolt, B. Slomski, A. Schilling, R. J. Cava, J. Osterwalder, and J. H. Dil, Phys. Rev. B 88, 035407 (2013).

[41] J. Sánchez-Barriga, M. R. Scholz, E. Golias, E. Rienks, D. Marchenko, A. Varykhalov, L. V. Yashina, and O. Rader, Phys. Rev. B 90, 195413 (2014).

[42] L. Bao, L. He, N. Meyer, X. Kou, P. Zhang, Z. Chen, A. V. Fedorov, J. Zou, T. M. Riedemann, T. A. Lograsso, K. L. Wang, G. Tuttle, and F. Xiu, Sci. Rep. 2, 726 (2012). 